\title{
Improved Student Success in Online Video-Supported Face-to-Face Lectures
}

\section{Prof. Ismail Uysal, University of South Florida}

Dr. Ismail Uysal has a Ph.D. in Electrical and Computer Engineering from the University of Florida. $\mathrm{He}$ is an assistant professor and the director of the RFID Lab for Applied Research at the University of South Florida. His research focuses on RFID and wireless sensors for supply chain, pharmaceuticals and healthcare as well as data analytics and machine learning applications for Internet-of-Things (IoT). His teaching focuses on improving student engagement especially for large classrooms and/or online sections. 


\title{
Improved Student Success in Online Video Supported Face-to-Face Lectures
}

\begin{abstract}
Traditionally, lectures can be divided into two main groups as far as content delivery is concerned. In face-to-face format, instructor delivers the content in the classroom in real time where students are expected to take notes to later refer to the course material when preparing for an exam. In the online format, the content is available as a lecture video and audio recording where the student can watch lectures on their own schedule. While face-to-face lectures have the advantage of enabling a more engaging atmosphere within the classroom, online lectures provide flexibility and archivability. Recently, flipped classrooms have gained in popularity by combining both methods to some extent and in different ways. However, the results reported in the literature so far in terms of student success are mixed. On the other hand, online lectures forego the traditional classroom time for media rich presentations of course material with inconclusive results. While there are other studies in the literature investigating the effects of video lectures on student understanding in traditional classrooms, they don't use recordings of actual classroom lectures. In this work however, face-to-face and online delivery methods are combined in a much more literal way by supporting traditional lectures with online video recordings of the same lectures which significantly reduces the additional teaching load of the instructor. Students are expected to attend face-to-face classes which are recorded and made available to them after the class for easy reference before the exams or to help reinforce difficult to learn topics. To compare changes in student success this study has been done throughout four academic semesters spanning a period of Spring 2014 to Fall 2016 with the same instructor, on a junior level "signals and systems" course for electrical engineers. The control classes had only traditional face-to-face lectures whereas the modified classes had online video support. No other differences existed in terms of course material, grading schemes, assignments, etc. In the end, statistically significant differences are observed in student grades between control sections and those supported with online video recordings. It is the author's opinion that repetition of course material in a way that doesn't affect time-in-class helped improve student success with minimal effort on the instruction side.
\end{abstract}

\section{Introduction}

The flipped classrooms have gained in popularity by proposing exciting methods to better prepare and engage the students throughout the content delivery process [1-4]. The effectiveness and student perception of flipped classrooms however, remains a topic of debate $[5,6]$. The biggest challenge of flipped classrooms is to ensure the students come prepared for the in-class lecture by reading/watching the (typically) online course material a prior. Based on the stateaction orientation theory of Kuhl [7], different students approach the pedagogy of flipped classrooms differently. For example, the action-oriented students benefit more from a flipped classroom approach via active engagement of course material outside the classroom and freediscussion environment within, whereas the state-oriented students prefer a more traditional way of content delivery where they can listen to the instructor and take notes as they've typically done since the elementary school. 
In this paper, we ask the simple question: "what if the students were provided with both options simultaneously, i.e., if we augmented a face-to-face, traditional class with online recordings of the same in-class lectures to create a content-repetitive environment reminiscent of a nonmandatory flipped-classroom approach?" In practical terms, each in-class lecture is recorded (as described in detail in the subsequent sections) and later made available to the students online for them to watch on-demand. The results show that the student success is improved significantly with little to no overhead in the instructional load.

\section{The proposed modification to traditional content delivery}

In this study, we propose an extremely basic modification to the way the course content is delivered for a traditional face-to-face lecture. More specifically, in the proposed method, faceto-face lectures are recorded during instruction, and later made available to the class in the form of online lecture recordings which the students can re-watch and review on-demand and on their own schedule.

During the control semester, the course content is delivered via face-to-face lectures and the student performance is assessed through conventional methods such as homework assignments, bi-weekly quizzes and midterm/final exams. During the test semesters, the same face-to-face lectures were recorded using a video management software (in this case, Panopto) which captures the screen to show the PowerPoint mark-ups while recording the instructor's voice (and any student questions/feedback) in real time. Figure 1 shows the viewing interface from the student's perspective with the lecture video playing on the right with each slide transition timeline marked for easy access.

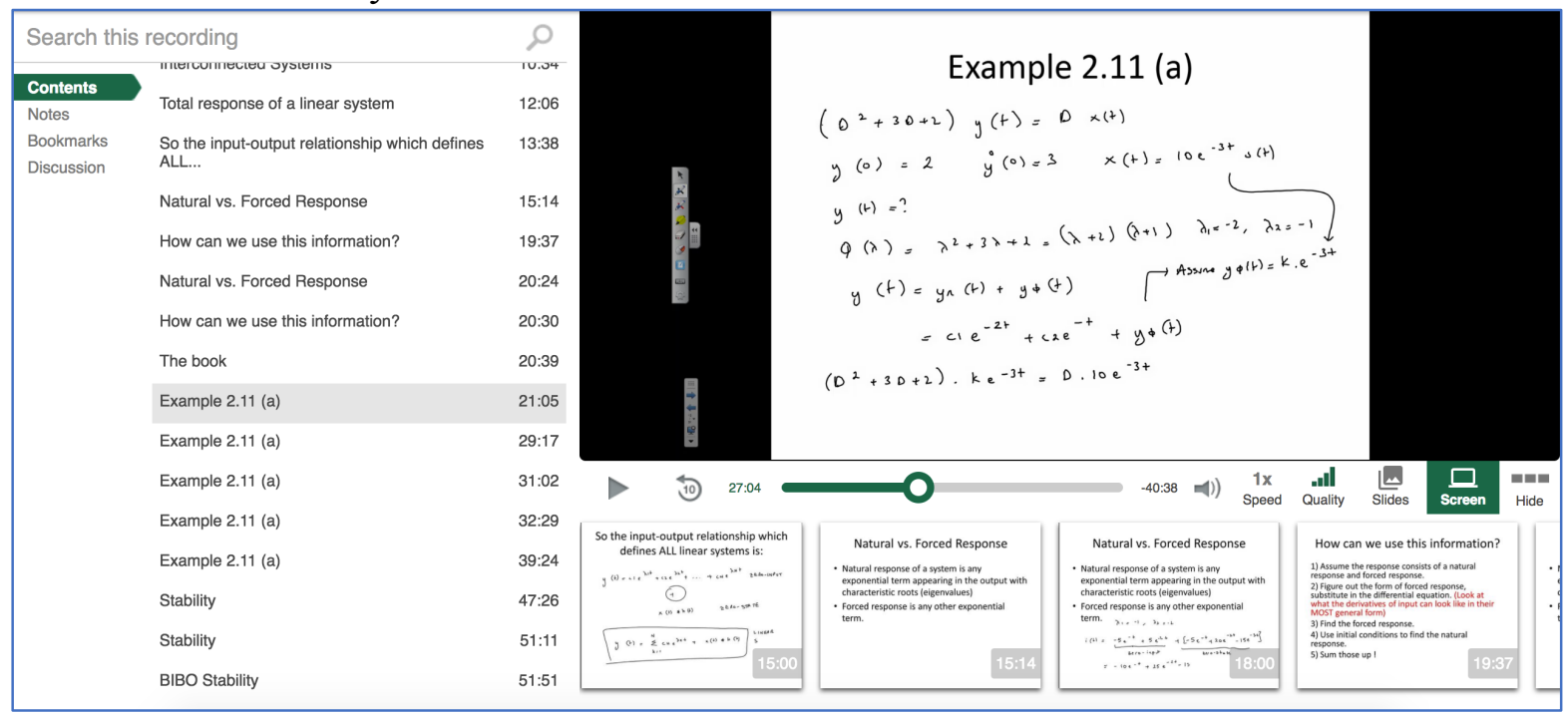

Figure 1. The Panopto user-interface which shows how the recorded face-to-face lectures are displayed for the students [8].

The learning management system used in this study is Canvas. It is important to note that the recorded lectures are made available on the course's Canvas webpage not just as a stand-alone video file, but in comprehensive learning modules which include slides, discussions as well as the recordings in a structured format as shown in figure 2. This format resembles almost a 
flipped classroom approach, yet the online content is simply a non-mandatory redelivery of the same face-to-face lectures.

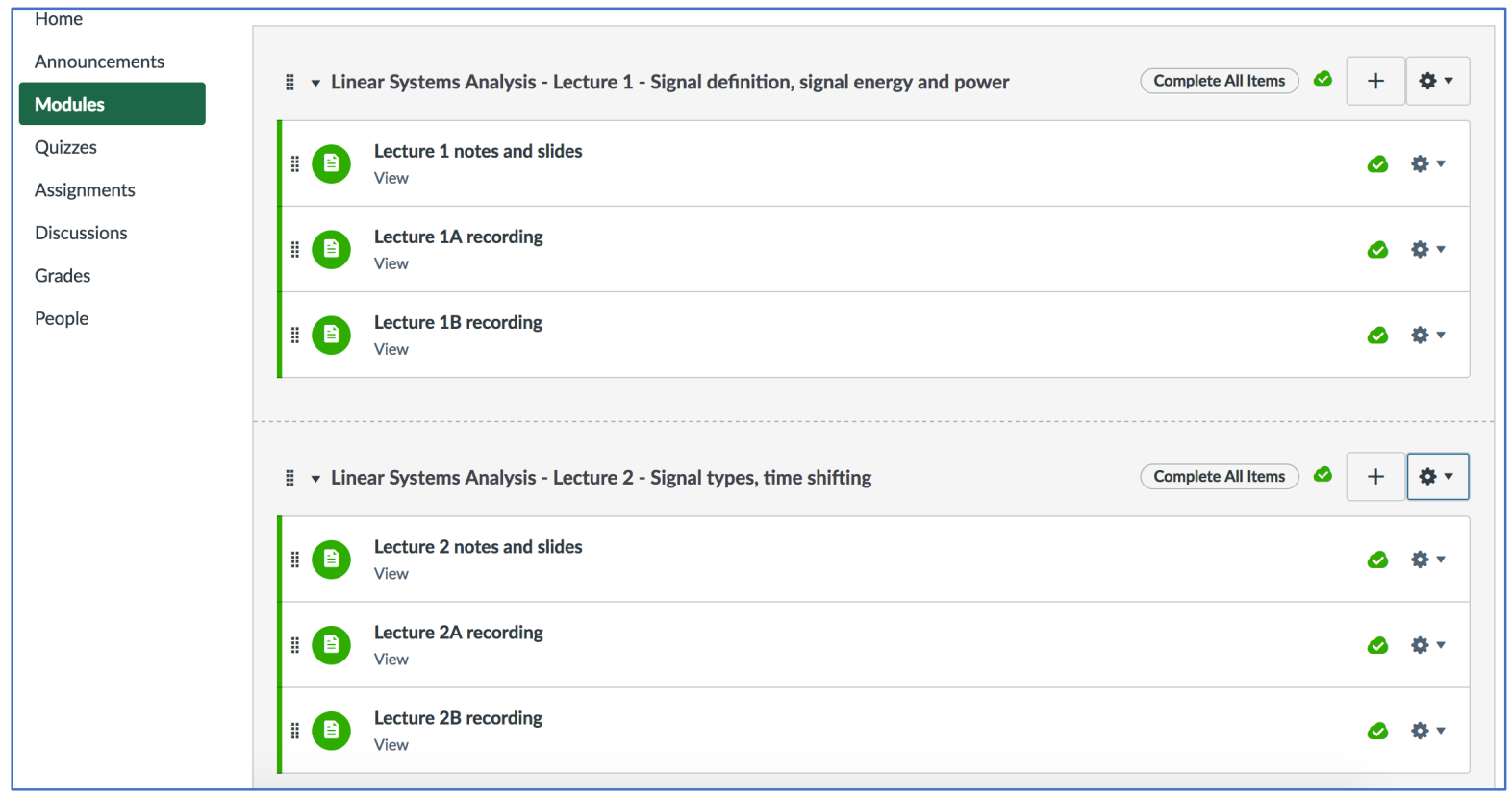

Figure 2. The Canvas user-interface which shows how the students can access the lecture recordings, notes and discussions online and on-demand.

\section{Methods and course/data specifics}

The course chosen for this study is a junior level course on signals and linear systems which is mandatory for all electrical engineering majors. The course goals include introducing the students to the fundamental concepts of signals and systems (both continuous and discrete time) while exploring different techniques to analyze linear systems using software tools and/or classical methods. The study has been carried out across four semesters which include two Spring and two Fall semesters to ensure some level of student uniformity in terms of possible semester-based performance differences. The online video support was not available throughout the control semester of Spring 2014. While the availability of the online video support was implemented during the second half of the Fall 2015 semester (before the $2^{\text {nd }}$ midterm exam), the lectures were available online since the beginning of the semester for Spring 2016 and Fall 2016 semesters. The course content and structure were identical between these four. In fact, other than the availability of online lecture recordings, no differences existed in terms of the instructor (same instructor taught all 4 semesters), grading schemes, quizzes, assignments, and most importantly, the exams.

The grade contributions for each assignment were as follows: i) $10 \%$ for homework assignments, ii) $10 \%$ for online quizzes assigned every other week, iii) $25 \%$ for the first midterm exam, iv) $25 \%$ for the second midterm exam and v) $30 \%$ for the final exam. Since the homework assignments concentrated on analytics using software tools such as Matlab (not covered in the recorded lectures) and the quizzes' main purpose was to gauge class attention (with conceptually easy questions), this article will analyze the effects of the proposed change using mainly the individual scores for the three exams, all of which are proctored with closed books/notes. 
Furthermore, some exams (but not all) included bonus points in the form of a bonus question $(+5$ or +10 bonus points), hence for an unbiased and scientific comparison, each grade for the exams with the bonus questions was scaled down to 100 points prior to calculating the cumulative grade for each student. The cumulative grade for each student was calculated as follows:

$$
\text { Grade }_{\text {cumulative }}=E_{1} \times \frac{100}{\operatorname{Total~}_{E_{1}}} \times 0.25+E_{2} \times \frac{100}{\operatorname{Total}_{E_{2}}} \times 0.25+E_{f} \times \frac{100}{\operatorname{Total~}_{E_{f}}} \times 0.3
$$

where $E_{1}, E_{2}$ and $E_{f}$ show the original exam grades for exam 1, 2 and the final exam respectively. The Total points show the total amount of points available for each exam including the +5 or +10 bonus points if available. This cumulative grade has a maximum ceiling of 80 percentage points. Finally, the average enrollment for the class across the four semesters was 38 students and none of the semesters had mandatory attendance policy.

Finally, it is important to note that there are other studies in the literature investigating the effects of video lectures on student understanding in traditional classrooms such as the famous study by Brecht and Ogilby [9]. However, there are multiple important differences such as the fact that they don't use recordings of actual classroom lectures and mostly rely on student reported statistics. In this study, face-to-face and online delivery methods are combined in a much more literal way by supporting traditional lectures with online video recordings of the very same lecture which significantly reduces the additional teaching load of the instructor. Furthermore, viewing statistics and analysis are done using the Panopto server for a more objective approach.

\section{Results and analysis}

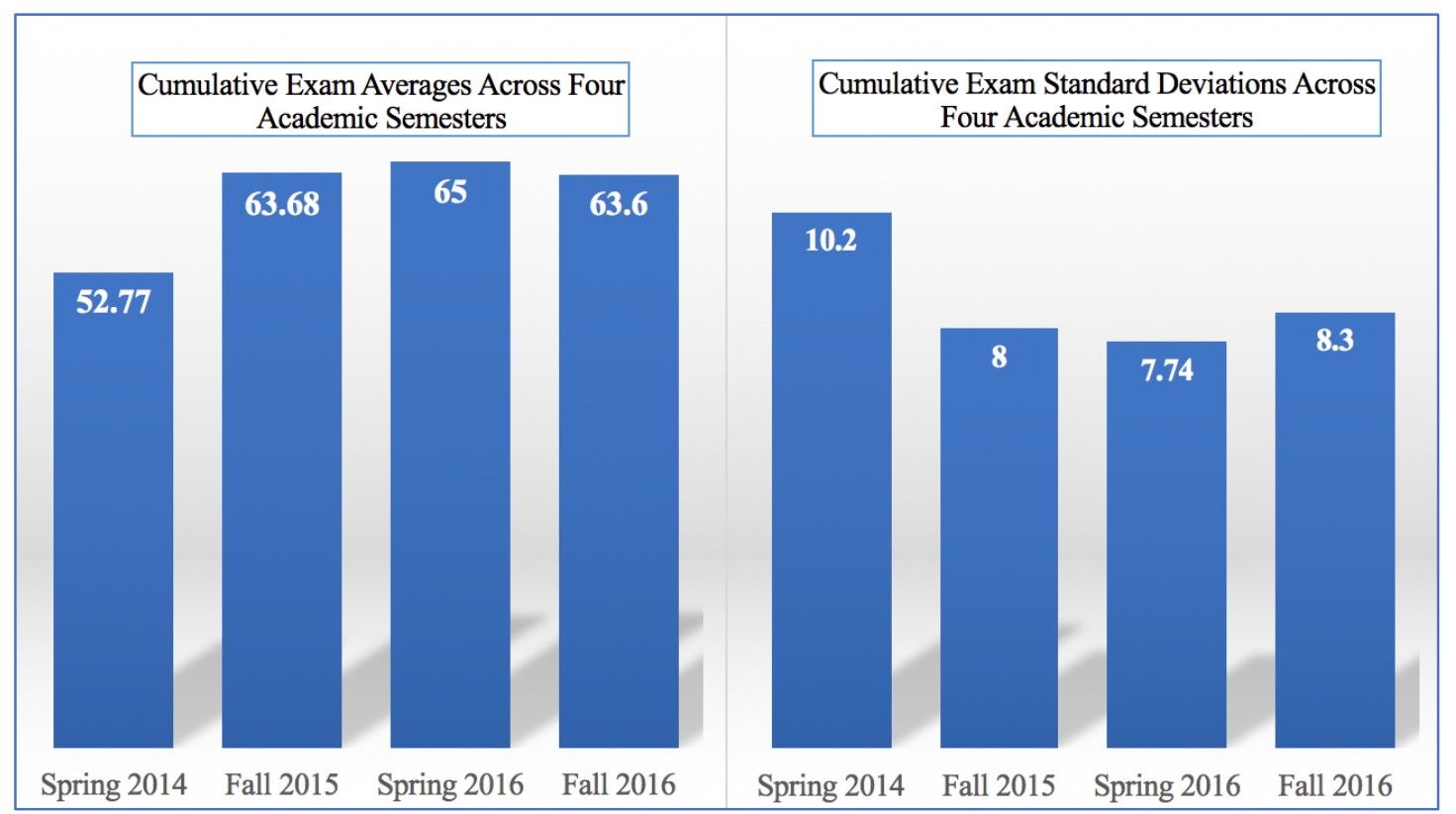

Figure 3. The means and standard deviations of the cumulative average exam grades for the four academic semesters as described in this study.

Figure 3 above shows the mean and the standard deviation of the cumulative grade (as calculated in equation (1) for the four semesters. As a reminder Spring 2014 is the control semester where 
no lectures were available online for later viewing. The other three semesters are test semesters where online recordings were available. Fall 2015 was the first semester where the online recordings were first introduced before the second exam of that semester. Spring and Fall 2016 had the lecture recordings fully available throughout the semester. As clearly seen from the figure there is a significant increase of as much as 23\% (when comparing Fall 2016 and Spring 2014 semesters) in the average cumulative grade between the control and test semesters where the lecture recordings were available. Furthermore, the standard deviation is reduced by approximately $20 \%$ for the test semesters compared to the control semester. One can conclude that both on average and in terms of distributed class performance, making the recorded in-class lectures available online, provides a valuable tool for improved student performance.

Figure 4 below provides a more detailed look at the distribution of the individual cumulative grades for the four semesters in the form of a radar plot. The innermost circle represents the control semester, Spring 2014 (a smaller radius indicates lower performance). One can see that the disparities in student performance mostly stem from the grade differences (more dominantly shown in the fourth quadrant) where a significantly more number of students scored between 20 to 60 points during Spring 2014 compared to the three test semesters. One interesting conclusion is that the availability of the online recordings for on-demand review of the course material might be helping the students who are struggling with the course material disproportionately more than the already successful students.

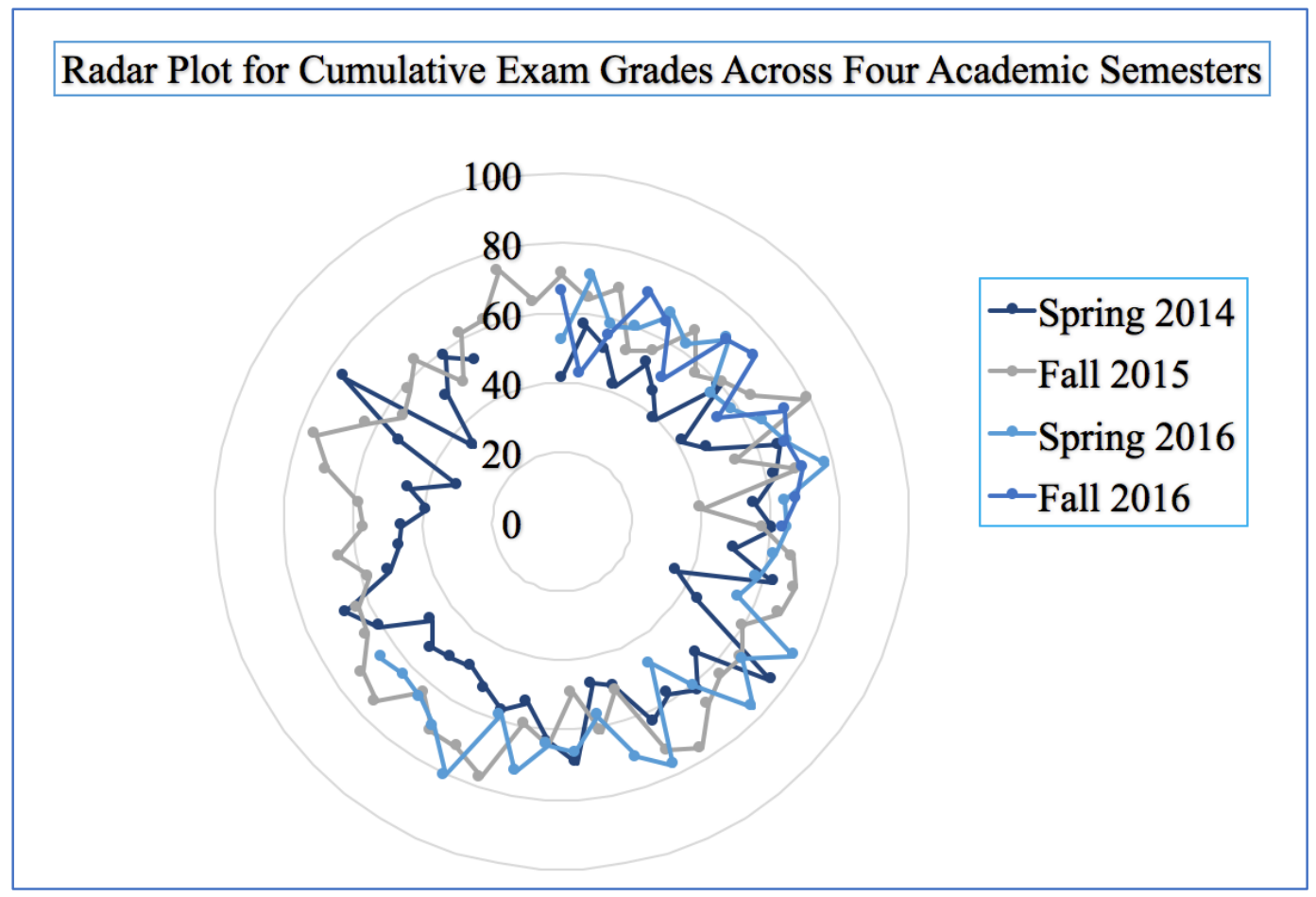

Figure 4. Radar plots for the cumulative average exam grades for each student enrolled during the four academic semesters as described in this study.

The cumulative average grades across the four semesters were compared using a two-sample ttest to observe if the control grade distribution is statistically significantly different than the test grade distribution. The result of the t-test decision confirms or rejects the null hypothesis that 
the data points in two different vectors (in this case the cumulative grade vectors calculated for control and test semesters) come from independent random samples from normal distributions with equal means and unknown variances. In this case, the outcome of the t-test was " 1 " when comparing the control semester to each of the three test semesters which means that the t-test rejected the null hypothesis at the 5\% significance level, indicating that the proposed modification resulted in statistically different and better grade distributions for the test semesters compared to the control semester. On the contrary, the outcome of the t-test was " 0 " when comparing each test semester with one another which means that the t-test did not reject the null hypothesis at the 5\% significance level, indicating statistically similar distributions between test semesters.

For additional insight, when one looks at the viewing statistics of the recorded lectures an interesting pattern emerges. To obtain the distribution of the percentage of unique students watching each lecture, the number of unique viewers is divided by the classroom enrollment for each of the 25 recorded lectures. Figure 5 shows both the distribution of the percentage of unique students watching each lecture as well as the distribution of the average minutes viewed per online lecture for Fall 2016 semester.
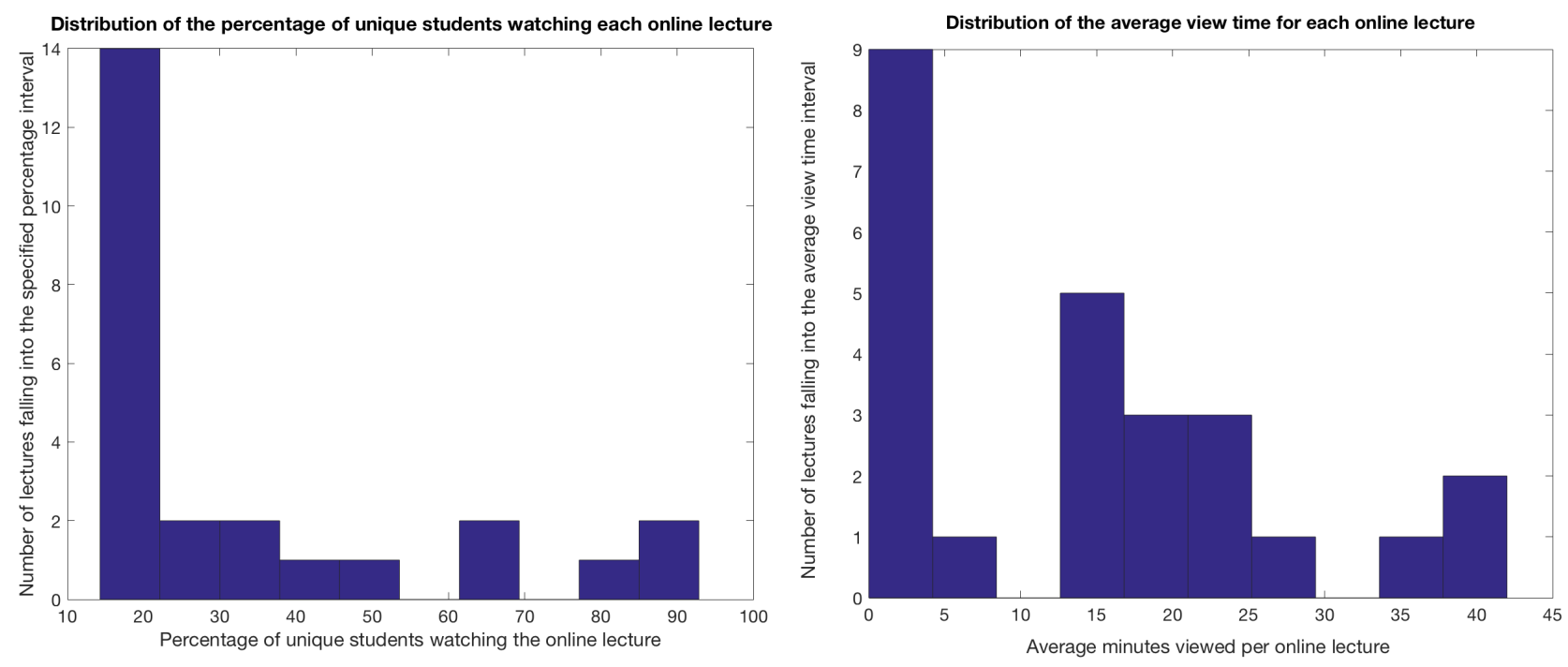

Figure 5. Distributions of percentage of unique students watching the lectures and average minutes watched per online lecture for Fall 2016 semester.

As one can see from the figure, both distributions are heavily skewed on the low end indicating that in general a lecture is watched by $\sim 10-20 \%$ of the class for an average of $\sim 5$ minutes (where each lecture is around 1 hour). These observations suggest that students watch lectures in short intervals focusing specifically on parts of the lecture where they had issues understanding. Furthermore, some lectures with conceptually more difficult topics (such as graphical convolution) have a higher percentage of students watching them for a longer period of time compared to other and more trivial lectures. 


\section{Conclusions and Future Work}

In this article, we present the results of a basic online modification to an otherwise traditional face-to-face electrical engineering lecture. The test semesters where each in-class lecture was recorded and posted on the course's Canvas webpage for later/on-demand viewing by the students, have demonstrated a statistically significant improvement in exam grades compared to the control semester with otherwise identical course content, delivery, layout, grading scheme and instructor. More importantly, the fact that this modification only requires minimal effort by the instructor, presents an easy, effective and obvious opportunity with a high return-on-timeinvestment to improve student success in a traditional classroom environment.

Future work will focus on conducting student surveys to collect specific feedback on their perception of these repetitive online recordings as well as a more detailed study of the video access statistics and how they change throughout the semester around exam times.

\section{References:}

[1] Bishop, J. L., \& Verleger, M. A. (2013, June). The flipped classroom: A survey of the research. In ASEE National Conference Proceedings, Atlanta, GA (Vol. 30, No. 9).

[2] Berrett, D. (2012). How'flipping'the classroom can improve the traditional lecture. The Education Digest, 78(1), 36.

[3] Fulton, K. (2012). Upside down and inside out: Flip your classroom to improve student learning. Learning \& Leading with Technology, 39(8), 12-17.

[4] Herreid, C. F., \& Schiller, N. A. (2013). Case studies and the flipped classroom. Journal of College Science Teaching, 42(5), 62-66.

[5] Strayer, J. F. (2007). The effects of the classroom flip on the learning environment: A comparison of learning activity in a traditional classroom and a flip classroom that used an intelligent tutoring system (Doctoral dissertation, The Ohio State University).

[6] Mason, G. S., Shuman, T. R., \& Cook, K. E. (2013). Comparing the effectiveness of an inverted classroom to a traditional classroom in an upper-division engineering course. IEEE Transactions on Education, 56(4), 430-435.

[7] Kuhl, J. (1985). Volitional mediators of cognition-behavior consistency: Self-regulatory processes and action versus state orientation. In Action control (pp. 101-128). Springer Berlin Heidelberg.

[8] Toppin, I. N. (2011). Video lecture capture (VLC) system: A comparison of student versus faculty perceptions. Education and Information Technologies, 16(4), 383-393.

[9] Brecht, H. D., \& Ogilby, S. M. (2008). Enabling a comprehensive teaching strategy: Video lectures. Journal of Information Technology Education, 7, 71-86. 